\title{
Effect of symmetry reduction on the spin dynamics of (001)-oriented GaAs quantum wells
}

\author{
D. J. English, ${ }^{1}$ J. Hübner, ${ }^{1}$ P. S. Eldridge, ${ }^{2}$ D. Taylor, ${ }^{3}$ M. Henini,,${ }^{3, *}$ R. T. Harley, ${ }^{4}$ and M. Oestreich ${ }^{1}$ \\ ${ }^{1}$ Institute for Solid State Physics, Leibniz University of Hannover, Appelstr. 2, 30167 Hannover, Germany \\ ${ }^{2}$ Foundation for Research and Technology, IESL, PO Box 1527, 71110, Heraklion, Crete, Greece \\ ${ }^{3}$ School of Physics and Astronomy, University of Nottingham, Nottingham, NG7 4RD, United Kingdom \\ ${ }^{4}$ School of Physics and Astronomy, University of Southampton, Southampton, SO17 1BJ, United Kingdom
}

(Received 21 December 2012; published 6 February 2013)

\begin{abstract}
Spin quantum beat spectroscopy is employed to investigate the in-plane anisotropy of the spin dynamics in (001) GaAs/AlGaAs quantum wells induced by an external electric field. This technique allows the anisotropy of the spin relaxation rate $\Gamma^{s}$ and the electron Landé $g$ factor $g^{*}$ to be measured simultaneously. The measurements are compared to similar data from (001) GaAs/AlGaAs quantum wells with applied shear strain and asymmetric barrier growth. All of these operations act to reduce the symmetry compared to that of a symmetric (001) quantum well in an identical manner $\left(\mathrm{D}_{2 d} \rightarrow \mathrm{C}_{2 v}\right)$. However, by looking at the anisotropy of both $\Gamma^{s}$ and $g^{*}$ simultaneously we show that the microscopic actions of these symmetry breaking operations are very different. The experiments attest that although symmetry arguments are a very useful tool to identify the allowed spin dependent properties of a material system, only a microscopic approach reveals if allowed anisotropies will manifest.
\end{abstract}

DOI: 10.1103/PhysRevB.87.075304

PACS number(s): 71.70.Ej, 73.21.Fg

\section{INTRODUCTION}

In recent decades there have been numerous proposals for devices that utilize the spin degree of freedom of electrons in semiconductors for spintronic applications. ${ }^{1-3}$ Heterostructures based upon III-V compounds are of particular interest in this field because they lack an inversion center which introduces a spin dependent splitting of the electron energy dispersion characterised by the spin-orbit field., ${ }^{4,5}$ The spin dynamics can be manipulated and controlled either by engineering the structure or via the application of external fields to enhance or attenuate the bulk crystal spin splitting. ${ }^{6,7}$ However, the microscopic mechanisms of these perturbations and their effects on the spin dynamics must be understood before full control can be achieved.

The spin-orbit field in semiconductors is a direct consequence of inversion asymmetry in the crystal structure. ${ }^{8}$ Therefore, gaining control of the symmetry of the crystal structure makes it possible to mold the spin dynamics as favored. A bulk zinc-blende crystal has the tetrahedral symmetry point group $T_{d}$ which is essentially isotropic. Therefore, the spin dynamics in bulk crystal are also isotropic with relation to orientation of the spins in the crystal lattice. Growth of a quantum well on a (001) zinc-blende substrate reduces the symmetry to the point group $D_{2 d} .{ }^{9}$ This point group possesses fourfold symmetry in the plane of the well, but with the symmetry broken between the in-plane $(x, y)$ and out-of-plane $(z)$ directions. The spin dynamics are therefore allowed to display anisotropy between these components. The symmetry of a (001) quantum well can be reduced further by the application of an electric field along the growth axis, shear strain in the plane of the well, or through asymmetric barrier growth. These perturbations act to remove the mirror symmetry of the quantum well and thus reduce the point group to $C_{2 v} .{ }^{9}$ This means that in addition to the anisotropy of the in-plane and out-of-plane components a twofold anisotropy in the plane of the well is allowed to develop. However, simply because anisotropy of the spin dynamics is allowed by symmetry considerations this does not imply that it will always occur. ${ }^{10,11}$
The reduction of the symmetry in (001) semiconductor quantum wells via the three methods under discussion here (electric field, shear strain, or asymmetric barriers) can affect the spin-orbit splitting and/or induce a gradient in the conduction and valence bands. While symmetry arguments inform us that anisotropy is allowed, the origin of the in-plane anisotropy of $\Gamma^{s}$ or $g^{*}$ lies in these microscopic actions. In (001) quantum wells, anisotropy of $\Gamma^{s}$ requires, in addition to the bulk inversion asymmetry term (BIA), another spin-orbit term of the same form as the structural inversion asymmetry (SIA) term, ${ }^{12}$ whereas for an anisotropic $g^{*}$ an asymmetric conduction electron wave function is needed. ${ }^{13}$ Therefore, simultaneous measurement of the anisotropy of $\Gamma^{s}$ and $g^{*}$ can be used as a powerful tool to directly reveal the microscopic effects on the structure. In Refs. 10 and 11 we have already utilized this method to show that asymmetric barrier growth generates a conduction band gradient (Fig. 1) that is isomorphous with respect to the valence band and that shear strain produces an SIA type term while the conduction band remains symmetric. The anisotropy of the spin relaxation rate produced by an external electric field has previously been investigated but the $g$ factor anisotropy was omitted from this study. ${ }^{14}$ In this paper we complete this missing link in this topic and simultaneously measure the anisotropy of both $\Gamma^{s}$ and $g^{*}$ produced when an electric field is applied along the growth axis of the quantum well.

We use spin quantum beat spectroscopy ${ }^{15,16}$ to investigate the spin dynamics of symmetric (001) quantum wells with a variable potential gradient produced by an external electric field applied along the growth axis. The sample contains two different quantum well widths which allows the effect of electron confinement energy on the spin dynamics to be studied. The method gives the spin relaxation rates for spin components along the growth axis, $\Gamma_{\|}^{s}$, and in the quantum well plane $\Gamma_{\perp}^{s}$, and also the in-plane Landé $g$ factor of the photo-excited conduction electrons. We find that a nonzero potential gradient produces significant in-plane anisotropy of both $\Gamma_{\perp}^{s}$ and $g^{*}$. 


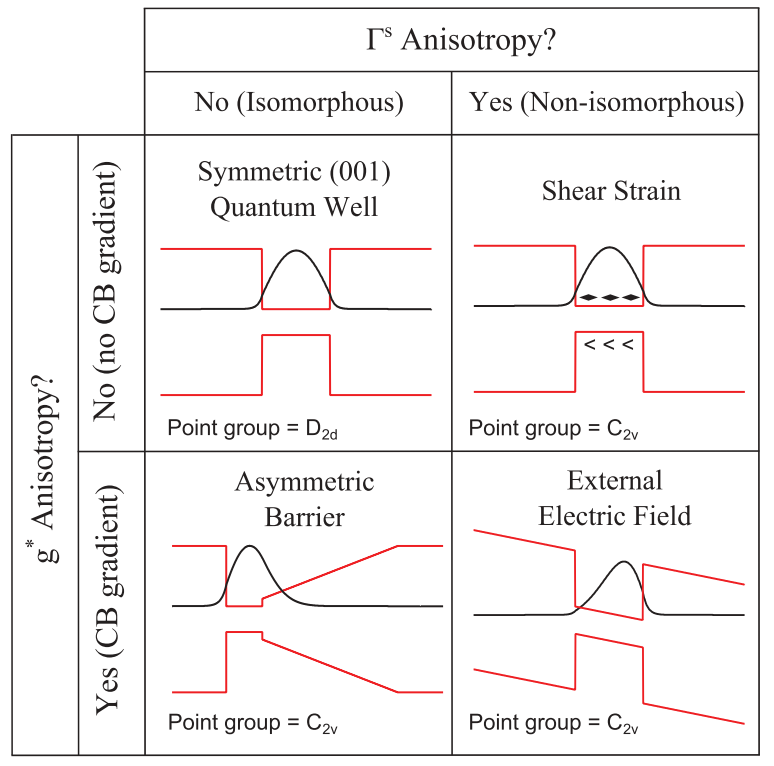

FIG. 1. (Color online) Table showing which symmetry breaking operations in (001) semiconductor quantum wells generate a conduction band gradient or an SIA type term using the anisotropy of the spin dynamics as an indicator.

\section{ORIGIN OF $\Gamma^{s}$ ANISOTROPY}

A detailed treatment of the origin of the anisotropy of the relaxation rate and $g$ factor is found in Refs. 10 and 11 . What follows is a summary of the important points. The dominant spin relaxation mechanism in (001) grown GaAs/AlGaAs quantum wells is the Dyakonov-Perel (DP) spin-dephasing mechanism. ${ }^{17}$ The electron experiences a momentum dependent effective magnetic field about which the electron spin precesses. Momentum relaxation occurs due to scattering from ionized impurities. This randomizes the momentum and hence the electron experiences a fluctuating effective magnetic field which causes spin polarization to be lost. ${ }^{18,19}$ The effective magnetic field is produced by inversion asymmetry in the structure of which there are three main sources that contribute to a total precession vector. These are the bulk inversion asymmetry from the host crystal $\Omega_{\mathbf{B I A}}$, the Rashba term $\Omega_{\text {SIA }}$, produced by a nonisomorphous gradient in the valence band, ${ }^{9}$ and the strain term $\Omega_{\text {STR }}$ from the application of shear strain. ${ }^{20}$ The resultant effective magnetic field is given as the sum of these terms such that $\Omega(\mathbf{p})=\Omega_{\text {BIA }}+\Omega_{\text {SIA }}+\Omega_{\text {STR }}$. The form of these terms and their momentum dependence are listed in Table I.

An SIA type term in the spin precession vector in a (001) zinc-blende quantum well interferes with the BIA term and thereby generates an anisotropy of the spin relaxation rate. ${ }^{12}$ This interference can produce an enhanced spin precession vector along one axis while canceling the vector along the perpendicular axis. The SIA term and the shear strain term have the same form, as indicated in Table I, and therefore either of these terms will cause the same interference. The SIA term is produced by a gradient of the valence band that is not proportional to the conduction band gradient, i.e., a nonisomorphous gradient. ${ }^{10,21}$ Therefore, the presence of in-plane relaxation rate anisotropy reveals the presence of either shear strain or a nonisomorphous valence band gradient.
TABLE I. Contributions to the spin precession vector to lowest order in electron momentum due to the BIA, SIA, and strain terms for a (001) quantum well. Where $\alpha, \gamma$, and $C_{3}$ are the Rashba, Dresselhaus, and strain coefficients respectively, $\epsilon_{i j}$ is the shear component of the strain tensor, and $p_{x, y, z}$ are the components of the electron momentum.

\begin{tabular}{cc}
\hline \hline $\begin{array}{c}\Omega(\mathbf{p}) \text { Term } \\
{[\mathrm{x}][\mathrm{y}][\mathrm{z}]}\end{array}$ & $\begin{array}{c}\text { Contribution } \\
{[100][010][001]}\end{array}$ \\
\hline$\Omega_{\text {BIA }}$ & $\frac{\beta}{\hbar^{2}}\left\{-p_{x}, p_{y}, 0\right\}$ \\
$\Omega_{\text {SIA }}$ & $\frac{\alpha}{\hbar^{2}}\left\{p_{y},-p_{x}, 0\right\}$ \\
$\Omega_{\text {STR }}$ & $\frac{C_{3}}{\hbar^{2}} \epsilon_{x y}\left\{p_{y},-p_{x}, 0\right\}$ \\
\hline \hline
\end{tabular}

Using the spin precession vectors in Table I, the spin relaxation rate projected onto the $z$ axis can be derived as a function of $\theta$, which is defined as the angle that an in-plane magnetic field makes to the [110] axis. ${ }^{14}$ This gives

$$
\begin{aligned}
\Gamma^{s}(\theta) & =\frac{1}{2}\left[\Gamma_{\|}^{s}+\Gamma_{\perp}^{s}\left(\theta+\frac{\pi}{2}\right)\right] \\
& =\frac{3 C \beta^{2}}{4}\left[1+\left(\frac{\alpha}{\beta}\right)^{2}+\frac{2 \alpha}{3 \beta} \cos (2 \theta)\right],
\end{aligned}
$$

where $\Gamma_{\|}^{s}$ and $\Gamma_{\perp}^{s}$ are the in-plane and out-of-plane spin relaxation rates, respectively. The coefficient for the BIA term is given as

$$
\beta=\left\langle p_{z}^{2}\right\rangle \gamma / \hbar^{2},
$$

where $\gamma$ is the Dresselhaus coefficient and $\left\langle p_{z}^{2}\right\rangle$ is the expectation value of the momentum squared in the growth direction $z$. The momentum squared expectation value can be calculated using the relation $\left\langle p_{z}^{2}\right\rangle=E_{1} \cdot m^{*}$, where $E_{1}$ is the energy of the lowest subband and $m^{*}$ is the electron effective mass. The coefficient for the SIA term is

$$
\alpha=\alpha^{\prime} e E_{z},
$$

where $\alpha^{\prime}$ is the Rashba coefficient, $e$ is the charge of an electron, and $E_{z}$ is the electric field in the $z$ direction. The coefficient $C$ is a constant derived from the momentum scattering time.

\section{ORIGIN OF $g^{*}$ ANISOTROPY}

A gradient of the conduction band in the growth direction causes the conduction electron wave function to become asymmetric. When an external magnetic field is applied in the plane of the well there are small corrections to the electron momentum due to Lorentz motion along the $z$ axis. Due to the asymmetry of the wave function these additional terms are not averaged out and thus small corrections to the Hamiltonian are produced. As demonstrated by Kalevich and Korenev, these corrections introduce off-diagonal elements into the $g$ factor tensor, which therefore has three independent components, i.e., $g_{x x}=g_{y y}, g_{z z}$, and $g_{x y}=g_{y x} \neq 0 .{ }^{13}$ This yields an in-plane anisotropy of the $g$ factor which is given by ${ }^{10,13,22}$

$$
g(\theta)=-\sqrt{g_{x x}^{2}+g_{x y}^{2}+2 g_{x x} g_{x y} \cos (2 \theta)},
$$

where $\theta$ is the angle of the magnetic field in the quantum well plane with respect to the [110] direction. The value of $g_{x x}$ is 
negative for quantum wells that are wider than $\approx 7 \mathrm{~nm}$, as is the case for the quantum wells investigated in this report. ${ }^{23,24}$ The magnitude of the off diagonal element is given as ${ }^{13}$

$$
g_{x y}=g_{y x}=\left(\frac{2 \gamma e}{\hbar^{3} \mu_{B}}\right)\left(\left\langle p_{z}^{2}\right\rangle\langle z\rangle-\left\langle p_{z}^{2} z\right\rangle\right),
$$

where \langle\rangle denotes an expectation value over the electron wave function. The asymmetry of the electron wave function in the conduction band is defined by the term $\left(\left\langle p_{z}^{2}\right\rangle\langle z\rangle-\left\langle p_{z}^{2} z\right\rangle\right)$ in Eq. (5). This term will be zero when the electron wave function is symmetrical with respect to reflection in a plane perpendicular to the growth direction. Therefore, in-plane anisotropy of $g^{*}$ for [001]-grown quantum wells is a consequence of asymmetry of the conduction band electron wave function and is modulated by the Dresselhaus coefficient $\gamma$.

\section{EXPERIMENT}

The sample investigated was a p-i-n heterostructure with the insulating region containing undoped, (001)-oriented, $\mathrm{GaAs} / \mathrm{Al}_{0.4} \mathrm{Ga}_{0.6} \mathrm{As}$ quantum wells of two different widths $(17.5 \mathrm{~nm}$ and $10 \mathrm{~nm})$ with five repeats of each width of quantum well and $14 \mathrm{~nm}$ barriers between the wells. The p-i-n heterostructure allowed the application of an easily controllable and homogeneous potential gradient. The sample was mounted on a rotation stage in a liquid helium cryostat with a variable superconducting magnet reaching transverse fields of up to $8 \mathrm{~T}$. The growth axis of the sample coincided with the direction of excitation and the rotation axis of the mount. The axis of the horizontal magnetic field was aligned perpendicular to the growth axis.

The technique of spin quantum spectroscopy was employed to measure the time resolved spin dynamics as described in Refs. 4 and 16. The sample was excited with circularly polarized picosecond laser pulses incident parallel to the growth direction by a mode locked $80 \mathrm{MHz}$ Ti:Sapphire laser oscillator at a wavelength of $750 \mathrm{~nm}$. The laser light was focused onto the sample yielding an excitation density of $\approx 2 \times 10^{8} \mathrm{~cm}^{-2}$. A liquid crystal retarder and a polarizer were used to detect the two circular polarization components of the excited photoluminescence (PL) consecutively. The intensity of both components were recorded using a synchroscan streak camera imaging system which spectrally and temporally resolved the PL with a resolution of $0.5 \mathrm{~nm}$ and $8 \mathrm{ps}$, respectively.

Absorption of circularly polarized light creates spin polarized populations of electrons and holes whose momentum distribution rapidly thermalizes after excitation via the emission of phonons and other scattering events. The hole population loses its spin orientation on the time scale of momentum relaxation $\left(\tau_{p}^{*}<1 \mathrm{ps}\right)$ due to strong valence band mixing and momentum dependent spin splitting. The degree of circular polarization of the PL, $P_{\sigma}=\left(\sigma^{+}-\sigma^{-}\right) /\left(\sigma^{+}+\sigma^{-}\right)$, is thus proportional to the conduction electron spin polarization. ${ }^{16}$

\section{RESULTS AND DISCUSSION}

Figure 2 shows the time evolution of the component of the spin polarization parallel to the $z$ axis for both well widths. Both data sets were measured simultaneously at a sample

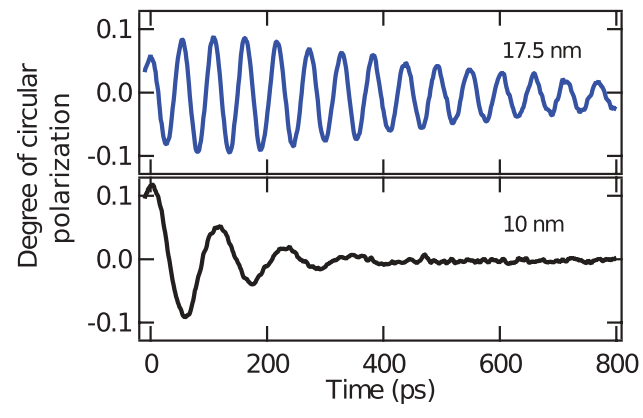

FIG. 2. (Color online) The time evolution of the degree of circular polarization for the two different well widths, $17.5 \mathrm{~nm}$ (top) and $10 \mathrm{~nm}$ (bottom), at a sample temperature of $25 \mathrm{~K}$.

temperature of $25 \mathrm{~K}$ for a reverse bias of $0 \mathrm{~V}$ and a magnetic field of $4 \mathrm{~T}$ applied parallel to the in-plane [110] axis. All the measurements we report here were conducted at $25 \mathrm{~K}$ which insured a high PL intensity and therefore a good signal to noise ratio. The observed oscillation of the degree of circular polarization is caused by the coherent Larmor precession of the conduction electron spin population with frequency $\omega_{L}=g^{*} \mu_{B} B / \hbar$ about the external magnetic field $B$. In order to extract both the spin relaxation rate $\Gamma^{s}$ and the $g$ factor the data was fitted to $A \cdot \cos \left(\omega_{L} t\right) e^{-\Gamma^{s} t}$ with $A, \omega_{L}$, and $\Gamma^{s}$ as free parameters.

Quantum confinement acts to decrease the absolute magnitude of the effective $g$ factor compared to that of the bulk value. ${ }^{9}$ For bulk GaAs the effective $g$ factor is negative and hence an increasing confinement energy causes $g_{x x}$ to tend back towards the free electron Landé $g$ factor. Furthermore, increasing confinement along the $z$ axis causes an increase in the spin relaxation rate since $\left\langle p_{z}^{2}\right\rangle$ and thereby the BIA coefficient $\beta$ increases. Therefore, the two well widths have different values of $g^{*}$ and $\Gamma^{s}$ as can be seen easily in Fig. 2 . The $17.5 \mathrm{~nm}$ quantum well signal has a spin lifetime of $411 \mathrm{ps}$ and a $g$ factor of -0.325 whilst the $10 \mathrm{~nm}$ quantum well signal has has a spin lifetime of $120 \mathrm{ps}$ and a $g$ factor of -0.155 .

Figure 3 displays the variation of both $g^{*}$ (open circles) and $\Gamma^{s}$ (closed circles) as a function of the in-plane angle of the magnetic field $B$ to the [110] crystal axis. Figure 3(a) displays the data for the $10 \mathrm{~nm}$ well with an applied reverse bias of $2 \mathrm{~V}$, which corresponds to the maximum anisotropy of both $\Gamma^{s}$ and $g^{*}$. Figure 3(b) displays the data for the $17.5 \mathrm{~nm}$ well with an applied reverse bias of $0.8 \mathrm{~V}$, corresponding to the maximum relaxation rate anisotropy reaching the particular condition where the Dresselhaus and Rashba splitting are equal and cancel each other for distinct points in momentum space, i.e., $\alpha / \beta \approx 1$. The solid lines indicate fits to Eqs. (1) and (4) which give values of $\alpha / \beta, g_{x y}$, and $g_{x x}$ for different values of applied field.

The extracted values of $g_{x y}$ are shown in Fig. 4 as a function of reverse bias for both the $10 \mathrm{~nm}$ (solid circles) and the $17.5 \mathrm{~nm}$ (open circles) well widths. The solid lines are numerically calculated values for $g_{x y}$ using the asymmetry term $\left(\left\langle p_{z}^{2}\right\rangle\langle z\rangle-\left\langle p_{z}^{2} z\right\rangle\right)$ in Eq. (5) with a fixed Dresselhaus splitting of $\gamma=11 \mathrm{eV} \AA^{3}$. The value of $\gamma$ perfectly fits previous measurements on two dimensional heterostructures ${ }^{25}$ and as suggested in Ref. 26 does not change with confinement. Therefore, the change in $g_{x y}$ can be unambiguously attributed 


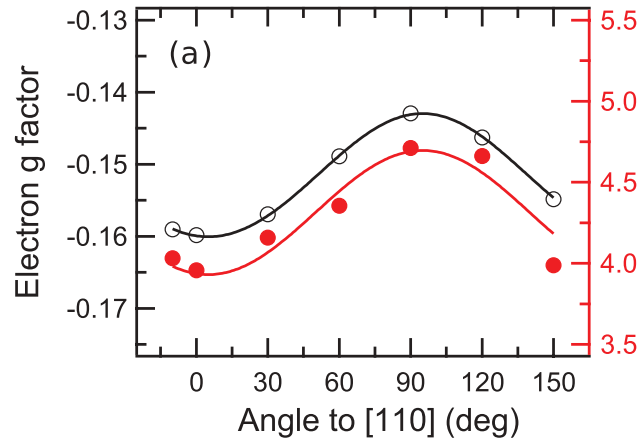

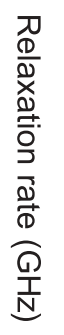
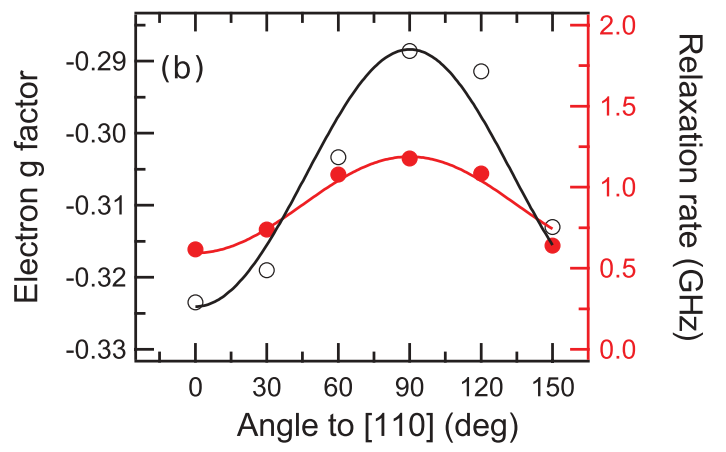

FIG. 3. (Color online) The variation of both the effective $g$ factor (open circles) and relaxation rate (closed circles) are displayed as a function of the angle of the magnetic field $B$ to the [110] axis at a temperature of $25 \mathrm{~K}$ for (a) the $10 \mathrm{~nm}$ quantum well with a negative bias of $2 \mathrm{~V}$ applied and (b) the $17.5 \mathrm{~nm}$ quantum well with a negative bias of $0.8 \mathrm{~V}$ applied.

to the magnitude of the asymmetry of the electron wave function due to the electric field.

The value of the ratio of $\alpha / \beta$ is obtained by fitting the spin relaxation rate in Fig. 3 to Eq. (1). The value of $\beta$ is calculated according to Eq. (2) with $\gamma=11 \mathrm{eV} \AA^{3}$, as given in Ref. 26, and the numerically obtained values of $\left\langle p_{z}^{2}\right\rangle$, which are dependent on the electric field. From the individual values of $\alpha / \beta$ and $\beta$ one obtains the absolute value of $\alpha$. Figure 5 shows the extracted values of $\alpha$ plotted versus the applied reverse bias

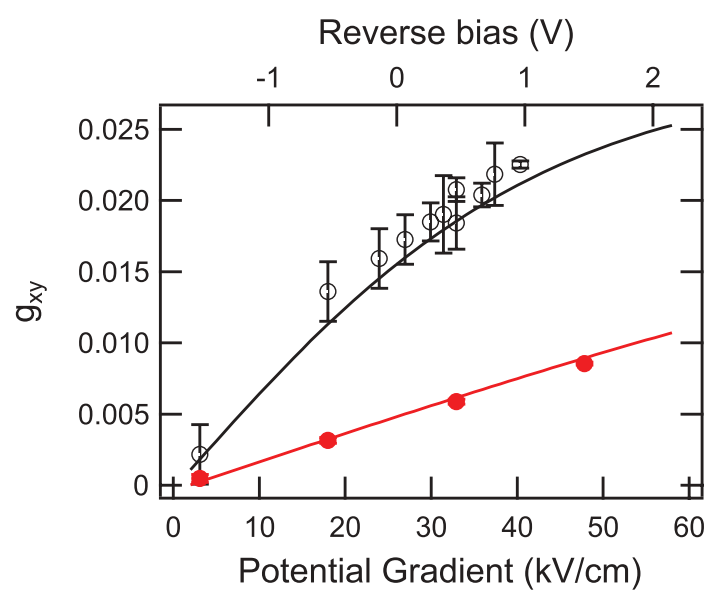

FIG. 4. (Color online) Variation of $g_{x y}$ as a function of the potential gradient for the $10 \mathrm{~nm}$ quantum well (solid circles) and the $17.5 \mathrm{~nm}$ quantum well (open circles). Solid lines show the calculated values of $g_{x y}$ for a fixed Dresselhaus constant for these well widths.

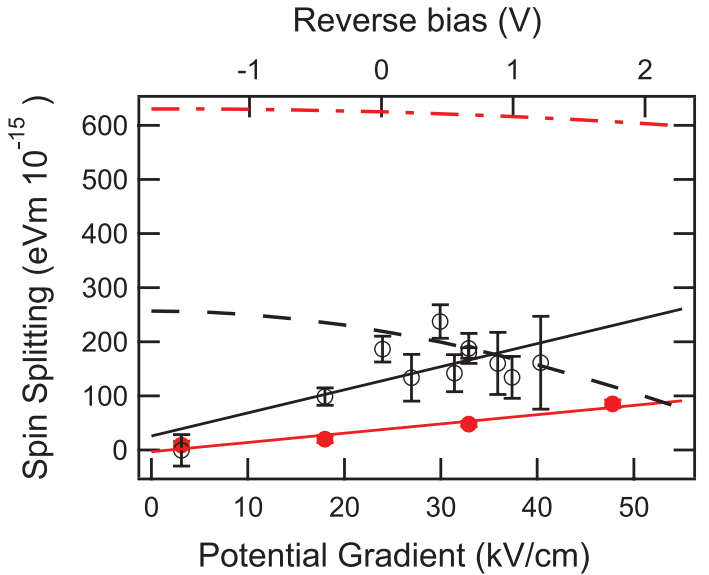

FIG. 5. (Color online) Dependence of $\alpha$ as a function of the potential gradient induced by an electric field parallel to the growth direction for the $10 \mathrm{~nm}$ quantum well (solid circles) and the $17.5 \mathrm{~nm}$ quantum well (open circles). Solid lines show linear fits to the data. The numerically calculated values of $\beta$ are displayed as a function of electric field for both the $17.5 \mathrm{~nm}$ (dashed line) and $10 \mathrm{~nm}$ (dot-dashed line) quantum wells. The values of $\alpha$ and $\beta$ for the $17.5 \mathrm{~nm}$ wells intercept at $\approx 35 \mathrm{kV} / \mathrm{cm}$ corresponding to $\alpha / \beta=1$.

for the $17.5 \mathrm{~nm}$ (open circles) and the $10 \mathrm{~nm}$ (solid circles) well widths. The numerically calculated values of $\beta$ are also shown as a function of electric field for both the $17.5 \mathrm{~nm}$ (dashed line) and $10 \mathrm{~nm}$ (dot-dashed line) quantum wells. The values of $\alpha$ and $\beta$ cross at a potential gradient of $35 \mathrm{kV} / \mathrm{cm}$ which corresponds to $\alpha / \beta=1$. As expected from Eq. (3), $\alpha$ is linearly dependent on the external electric field. The solid lines are linear fits to this data with the gradient giving the values for the Rashba coefficient $\alpha^{\prime}$. These are $0.042 \mathrm{~nm}^{2}$ for the $17.5 \mathrm{~nm}$ quantum wells and $0.017 \mathrm{~nm}^{2}$ for the $10 \mathrm{~nm}$ quantum wells. These values are comparable in magnitude to previous measurements and clearly show a dependence on confinement energy in line with the energy dependence previously measured, i.e., larger confinement energy results in a smaller Rashba coefficient. ${ }^{25}$

A higher potential gradient lowers the effective confinement via the quantum confined Stark effect and increases the asymmetry of the wave function. Figures 4 and 5 show that increasing confinement energy significantly reduces the anisotropy of both $g^{*}$ and $\Gamma_{\perp}^{s}$. The reduction of $g_{x y}$ with confinement is due to a decrease in the asymmetry term in Eq. (5) for larger confinement energy and hence lower potential gradient. $^{22}$ The strength of the Rashba term $\alpha$ is mediated by the asymmetry of the wave function which is larger for wider quantum wells for a given potential gradient. The value of $\beta$ is proportional to the confinement energy and is therefore larger for the $10 \mathrm{~nm}$ well than the $17.5 \mathrm{~nm}$ well and decreases for increasing potential gradient. Therefore, in wider quantum wells a smaller electric field is required to produce a value of $\alpha$ that is comparable to $\beta$. This is the case in the $17.5 \mathrm{~nm}$ quantum well where the application of only a modest external electric field produces a ratio of $\alpha / \beta=1$. This indicates a regime where there is full cancellation of the spin-orbit field along the [110] axis. The DP contribution to $\Gamma_{\|}^{s}$ for spins aligned perpendicular to this axis should vanish. The fact that the spin lifetime only increases to $2 \mathrm{~ns}$ indicates that other 
relaxation mechanisms play a role once DP is canceled as has been observed in other systems. ${ }^{4,27}$

\section{SUMMARY}

The measurement of the in-plane anisotropy of the spin dynamics in (001) quantum wells can be used as a tool to reveal the microscopic actions of different symmetry breaking perturbations on the band edges. We have investigated the anisotropy induced by asymmetric barrier growth in Ref. 10, the application of strain in Ref. 11, and the application of an external electric field in this paper. A comparison of the anisotropy produced by these three perturbations shows that while the change in symmetry is identical for each, the effects on the band edges are very different. An asymmetric potential produced by a graded alloy barrier induces $g$ factor anisotropy without anisotropy of spin relaxation. Therefore, the conduction band electron wave function must be asymmetric but the valence and conduction bands are isomorphous, i.e., related by a constant factor. ${ }^{10}$ Shear strain has the opposite effect and induces only a relaxation rate anisotropy while $g^{*}$ remains isotropic. Therefore, strain induces a spin splitting that can interfere with the bulk splitting while the wave function of the conduction electron remains symmetric. ${ }^{11}$ The work presented here reveals that an electric field applied along the growth axis produces a nonisomorphous gradient of the valence and conduction band and also causes the electron wave function to become asymmetric.

In conclusion, the effects on the symmetry of all three perturbations are identical and mean that the spin dynamics are allowed to develop an in-plane anisotropy. However, the appearance of anisotropy of $\Gamma^{s}$ or $g^{*}$ is not required, but is rather a consequence of the microscopic effect of the perturbation. Therefore, by simultaneously measuring the anisotropy of both quantities we have revealed the different microscopic actions of these symmetry breaking operations.

\section{ACKNOWLEDGMENTS}

We acknowledge the financial support of the DFG priority program "SPP 1285 - Semiconductor Spintronics," the BMBF joint research project "QuaHL-Rep," and the school "Contacts for Nanosystems" founded by the Niedersachsen Institutes of Technology (NTH).
*Also at: Nottingham Nanotechnology and Nanoscience Centre.

${ }^{1}$ S. A. Wolf, D. D. Awschalom, R. A. Buhrman, J. M. Daughton, S. von Molnár, M. L. Roukes, A. Y. Chtchelkanova, and D. M. Treger, Science 294, 1488 (2001).

${ }^{2}$ S. Datta and B. Das, Appl. Phys. Lett. 56, 665 (1990).

${ }^{3}$ I. Žutić, J. Fabian, and S. D. Sarma, Rev. Mod. Phys. 76, 323 (2004).

${ }^{4}$ M. I. Dyakonov, Spin Physics in Semiconductors, Springer Series in Solid-State Sciences (Springer, Berlin, 2008).

${ }^{5}$ F. Meier and B. P. Zakharchenya, Optical Orientation, Modern Problems in Condensed Matter Sciences, Vol. 8 (Elsevier, New York, 1984).

${ }^{6}$ G. L. Bir and G. E. Pikus, Symmetry and Strain-Induced Effects in Semiconductors (John Wiley and Sons, New York, 1974), p. 484.

${ }^{7}$ A. G. Aronov, G. E. Pikus, and A. N. Titkov, Sov. Phys. JETP 57, 680 (1983).

${ }^{8}$ M. Wu, J. Jiang, and M. Weng, Phys. Rep. 493, 61 (2010).

${ }^{9} \mathrm{R}$. Winkler, Spin-orbit Coupling Effects in Two-Dimensional Electron and Hole Systems, Springer Tracts in Modern Physics (Springer, Berlin, 2003).

${ }^{10}$ P. S. Eldridge, J. Hübner, S. Oertel, R. T. Harley, M. Henini, and M. Oestreich, Phys. Rev. B 83, 041301 (2011).

${ }^{11}$ D. J. English, P. G. Lagoudakis, R. T. Harley, P. S. Eldridge, J. Hübner, and M. Oestreich, Phys. Rev. B 84, 155323 (2011).

${ }^{12}$ N. S. Averkiev and L. E. Golub, Phys. Rev. B 60, 15582 (1999).

${ }^{13}$ V. K. Kalevich and V. L. Korenev, JETP Lett. 57, 571 (1993).

${ }^{14}$ A. V. Larionov and L. E. Golub, Phys. Rev. B 78, 033302 (2008).

${ }^{15}$ A. P. Heberle, W. W. Rühle, and K. Ploog, Phys. Rev. Lett. 72, 3887 (1994).
${ }^{16}$ M. Oestreich, S. Hallstein, and W. W. Ruhle, IEEE J. Sel. Top. Quantum Electron. 2, 747 (1996).

${ }^{17}$ A. Malinowski, R. S. Britton, T. Grevatt, R. T. Harley, D. A. Ritchie, and M. Y. Simmons, Phys. Rev. B 62, 13034 (2000).

${ }^{18}$ M. I. Dyakonov and V. I. Perel, Sov. Phys. Solid State 13, 3023 (1972).

${ }^{19}$ M. E. Flatte, J. M. Byers, and W. H. Lau, in Semiconductor Spintronics and Quantum Computation (Springer, Berlin, 2002), Chap. 4.

${ }^{20}$ G. E. Pikus, V. A. Marushchak, and A. N. Titkov, Sov. Phys. Semicond. 22, 115 (1988).

${ }^{21}$ P. S. Eldridge, W. J. H. Leyland, P. G. Lagoudakis, R. T. Harley, R. T. Phillips, R. Winkler, M. Henini, and D. Taylor, Phys. Rev. B 82, 045317 (2010).

${ }^{22}$ J. Hübner, S. Kunz, S. Oertel, D. Schuh, M. Pochwała, H. T. Duc, J. Förstner, T. Meier, and M. Oestreich, Phys. Rev. B 84, 041301 (2011).

${ }^{23}$ M. J. Snelling, G. P. Flinn, A. S. Plaut, R. T. Harley, A. C. Tropper, R. Eccleston, and C. C. Phillips, Phys. Rev. B 44, 11345 (1991).

${ }^{24}$ E. L. Ivchenko and A. A. Kiselev, Sov. Phys. Semicond. 26, 827 (1992).

${ }^{25}$ P. S. Eldridge, W. J. H. Leyland, P. G. Lagoudakis, O. Z. Karimov, M. Henini, D. Taylor, R. T. Phillips, and R. T. Harley, Phys. Rev. B 77, 125344 (2008).

${ }^{26}$ M. P. Walser, U. Siegenthaler, V. Lechner, D. Schuh, S .D. Ganichev, W. Wegscheider, and G. Salis, Phys. Rev. B 86, 195309 (2012).

${ }^{27}$ Y. Ohno, R. Terauchi, T. Adachi, F. Matsukura, and H. Ohno, Phys. Rev. Lett. 83, 4196 (1999). 